\title{
Thermal Marangoni Flow Impacts the Shape of Single Component Volatile Droplets on Thin, Completely Wetting Substrates
}

\author{
Samira Shiri $\oplus^{1,2}$ Shayandev Sinha $\odot,{ }^{1}$ Dieter A. Baumgartner, ${ }^{1}$ and Nate J. Cira $\odot^{1,2, *}$ \\ ${ }^{1}$ Rowland Institute, Harvard University, Cambridge, Massachusetts 02142, USA \\ ${ }^{2}$ Meinig School of Biomedical Engineering, Cornell University, Ithaca, New York 14853, USA
}

(Received 29 November 2020; accepted 3 May 2021; published 8 July 2021; corrected 17 August 2022)

\begin{abstract}
Despite surface energies dictating complete wetting, it has been classically observed that volatile alkanes do not spread completely on glass substrates, and faster evaporation rates lead to higher contact angles. Here we investigate how substrate thickness influences this behavior. For sufficiently thin substrates, we find alkanes evaporate slower and display higher apparent contact angles, at odds with the typical explanations involving just evaporation, capillarity, and viscous dissipation. We derive the droplet temperature distribution and use it as part of a criteria to show that thermal Marangoni contraction plays a significant role in establishing droplet shape on thin substrates.
\end{abstract}

DOI: 10.1103/PhysRevLett.127.024502

The interaction of liquid droplets with solid surfaces is ubiquitous in applications such as printing, natural resource extraction, microelectronics, and microfluidics. Even for simple liquids on simple surfaces, the presence of multiple physical effects, spanning molecular to macroscopic length scales, can give rise to an array of often surprising behaviors. For example, in the case of alkanes on clean glass, arguments based on surface energy predict that droplets should spread completely into a thin film $[1,2]$. Longer chain-length alkanes, such as hexadecane (Supplemental Material, Video V1 [3]) do spread continuously, however, the spreading dynamics are, counterintuitively, independent of the solid surface energy, explained by the capillary-driven advancement of the bulk droplet over a molecular-scale precursor film, slowed by viscous dissipation [7].

Shorter chain-length alkanes do not spread continuously but can exhibit pseudostable apparent contact angles and radii [Fig. 1(a), Supplemental Material, Video V2 [3]], seemingly in contradiction with simple energetic arguments for completely wetting surfaces. This behavior has been the topic of numerous studies, referenced nonexhaustively here [8-17]. The typical mechanism proposed to prevent spreading involves evaporation removing liquid fastest from the droplet perimeter, capillarity causing the droplet to spread, and viscous dissipation opposing flow within the droplet. Careful resolution of the singularities in viscous dissipation and evaporative flux at the droplet edge can result in

Published by the American Physical Society under the terms of the Creative Commons Attribution 4.0 International license. Further distribution of this work must maintain attribution to the author(s) and the published article's title, journal citation, and DOI. predictions of pseudostable droplet shapes [8-11,14]. Here, we refer to this explanation for pseudostable shapes as the direct evaporation-driven mechanism, Fig. 1(a).

Evaporation of volatile droplets can also cause nonuniform temperature and surface tension gradients that result in thermal Marangoni flow. A separate body of work has investigated such flows within single-component droplets on partially wetting substrates, often focusing on the flow
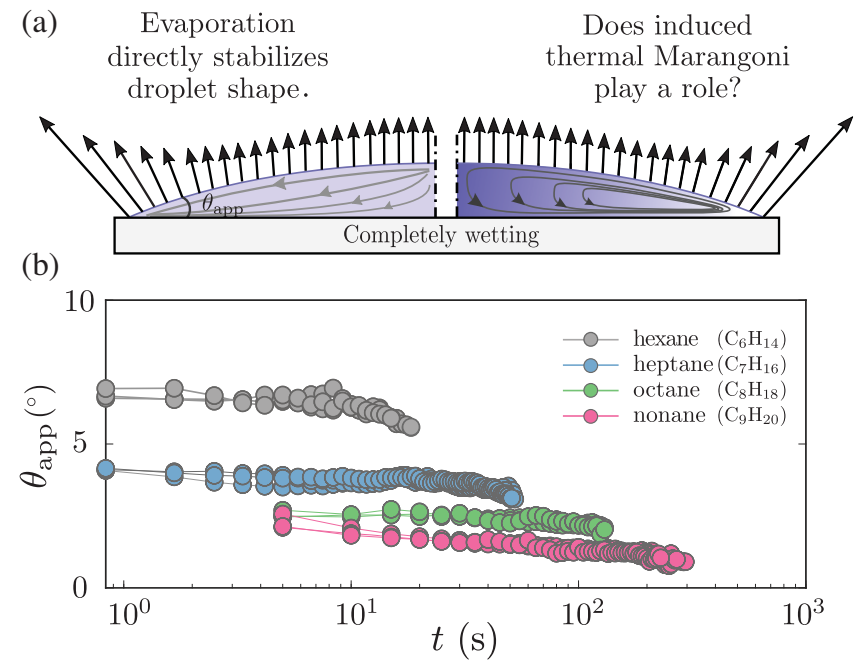

FIG. 1. Single component volatile liquids on a completely wetting surface. (a) Nonuniform evaporation is known to prevent droplet spreading through "direct evaporation-driven stabilization" by removing liquid fastest from the droplet perimeter. Here we ask, might Marangoni contraction from an inward surface tension-driven flow along the droplet-vapor interface play a role? (b) Apparent contact angle $\theta_{\text {app }}$ decreases with alkane chain length for hexane $(0)$, heptane $(0)$, octane $(0)$, and nonane (O) on glass (triplicate measurements shown). 
direction and its impact on deposition patterns during droplet drying. Experiments [18,19], asymptotic [20,21], and numeric $[22,23]$ approaches indicate that the resulting flows can be complex, pointing toward or away from the center of the droplet depending on contact angle, thermal conductivities of the liquid and solid, and substrate thickness.

Work on two-component droplets on high energy surfaces [24-28] has shown that solutal Marangoni flow directed from edge to center of a droplet can prevent droplet spreading by "Marangoni contraction," Fig. 1(a). We wondered if thermal Marangoni flows might play a role in preventing single-component droplets from spreading. Based on thermal conductivity [20], Marangoni flows for alkanes on glass should be in the correct direction for Marangoni contraction, but such thermal Marangoni flows are typically much weaker than solutal Marangoni flows. Most previous work investigating the shape of singlecomponent droplets on completely wetting surfaces has not included Marangoni effects, with some articles presenting scaling arguments to support this omission for volatile alkanes $\geq$ C6 [10,11,17], $\geq$ C7 [12], or $\geq$ C8 [9]. To the best of our knowledge, only one paper, Tsoumpas et al. [16], has included both direct evaporation-driven and thermal Marangoni effects in the study of a singlecomponent droplet on a completely wetting surface, finding that for volatile hydrofluoroethers Marangoni flows can significantly influence the droplet shape. Here we present experimental and theoretical results that help clarify the impact of thermal Marangoni flow on the spreading and shape of evaporating single-component droplets using the classic "alkanes on clean glass" system with substrates of different thickness.

We first sought to measure alkane droplet shapes on completely wetting substrates. Glass substrates were used immediately following cleaning by washing and corona treatment. $1.5 \mu \mathrm{L}$ droplets of hexane $\left(\mathrm{C}_{6} \mathrm{H}_{14}\right)$, heptane $\left(\mathrm{C}_{7} \mathrm{H}_{16}\right)$, octane $\left(\mathrm{C}_{8} \mathrm{H}_{18}\right)$, and nonane $\left(\mathrm{C}_{9} \mathrm{H}_{20}\right)$ were deposited and optical reflectometry [29] was used to measure the droplet shape. This technique is well suited for precise measurement of low angles and characterizes the angle created by the steepest section along the droplet interface, which we refer to here as the apparent contact angle (Supplemental Material, Sec. I A [3]).

For different alkanes, we find that the shorter the chain length, the higher the measured apparent contact angle and the faster the evaporation, Fig. 1(b). This trend is consistent with both previous experimental observations and the typical direct evaporation-driven stabilization explanation; shorter chain-length alkanes evaporate faster, and faster evaporation supports a larger apparent contact angle. However, since thermal Marangoni flows are also expected to increase with faster evaporation, it is difficult to categorically parse their role in determining droplet shape.
To more definitively establish whether Marangoni flow contributes to the droplet shape, we changed the substrate thickness. Temperature should be lower and evaporation thus slower on thin substrates, but, conveniently, inward Marangoni flow might be expected to increase [21]. We observe that the apparent contact angle for each alkane is higher and evaporation is slower on thinner substrates, Fig. 2 (Supplemental Material, Sec. I, Fig. S1 [3]), opposite
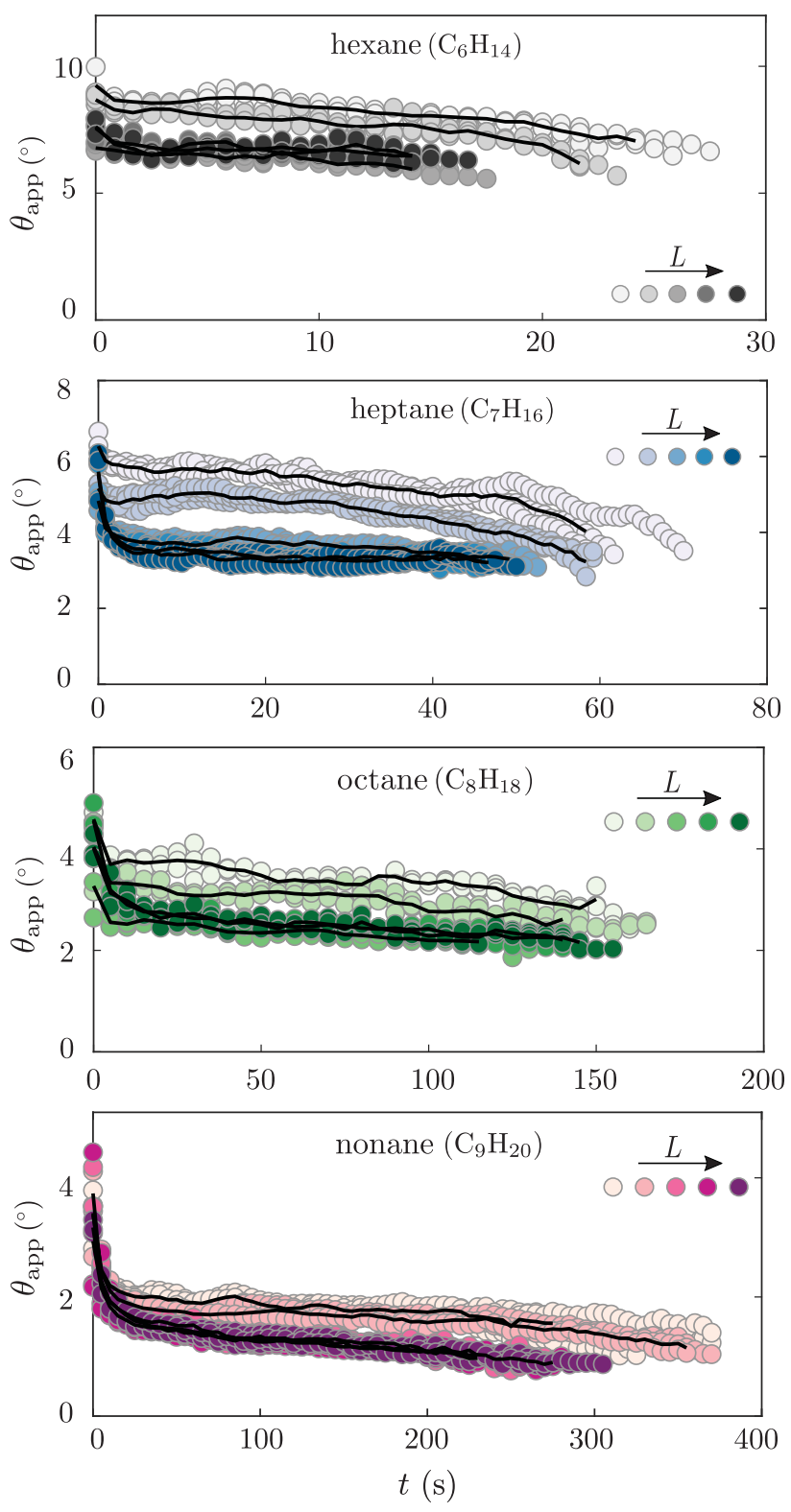

FIG. 2. Apparent contact angle $\theta_{\text {app }}$ of evaporating alkane droplets over time for different glass thicknesses $L$. The direct evaporation-driven mechanism proposed to stabilize droplets predicts higher angles for faster evaporation rates. C6-C9 alkanes on thinner glass show larger apparent contact angles and slower evaporation, suggesting the need to consider an additional effect determining droplet shape. Darker shades represent thicker glass substrates $(0.063,0.138,1,3$, and $6 \mathrm{~mm})$. 
the expectation from the direct evaporation-driven explanation. Though modest in absolute magnitude, the relative effect is nontrivial, typically a $80 \%-40 \%$ difference in apparent contact angle on thin substrates for all alkanes studied, suggesting a potential role for Marangoni effects in establishing droplet shape across thin substrates for all volatile alkanes. Interestingly, independent of alkane chain length, above $1 \mathrm{~mm}$ apparent contact angles were not changed by increasing the substrate thickness, suggesting Marangoni flow might play a negligible role in determining alkane droplets shapes when the substrate is thick.

To determine if internal flows were consistent with putative Marangoni contraction, we added and imaged fluorescent tracer particles in the droplets (Supplemental Material, Sec. I B [3]). We found, in all cases, Marangoni flow directed from the edge to center along the liquid-vapor interface balanced by a recirculating capillary flow. The magnitude of the flow was faster with shorter alkanes and thinner substrates [Fig. 3(a), Supplemental Material, Videos V2, V3, and V5 [3] ], consistent in direction and trend with a potential Marangoni contraction mechanism. Hexane had strong enough flows to form BénardMarangoni cells close to the center of the droplet, and flows in octane were slow enough that the tracer particles sedimented before fully circulating.

We next sought to theoretically predict the magnitude of thermal Marangoni flow to compare with experiments. To do this, we calculated the temperature distribution $T(r)$, Marangoni shear stress $\partial \gamma / \partial r=(\partial \gamma / \partial T)(\partial T / \partial r)$, and resulting Marangoni flow $u(r, z)$. For a sufficiently thin droplet, ignoring horizontal and convective heat transfer within the droplet and balancing evaporative heat loss and heat conduction through the liquid height gives

$$
\kappa_{\ell} \frac{T_{s}(r)-T_{d}(r)}{h(r)}=\mathcal{L} j(r),
$$
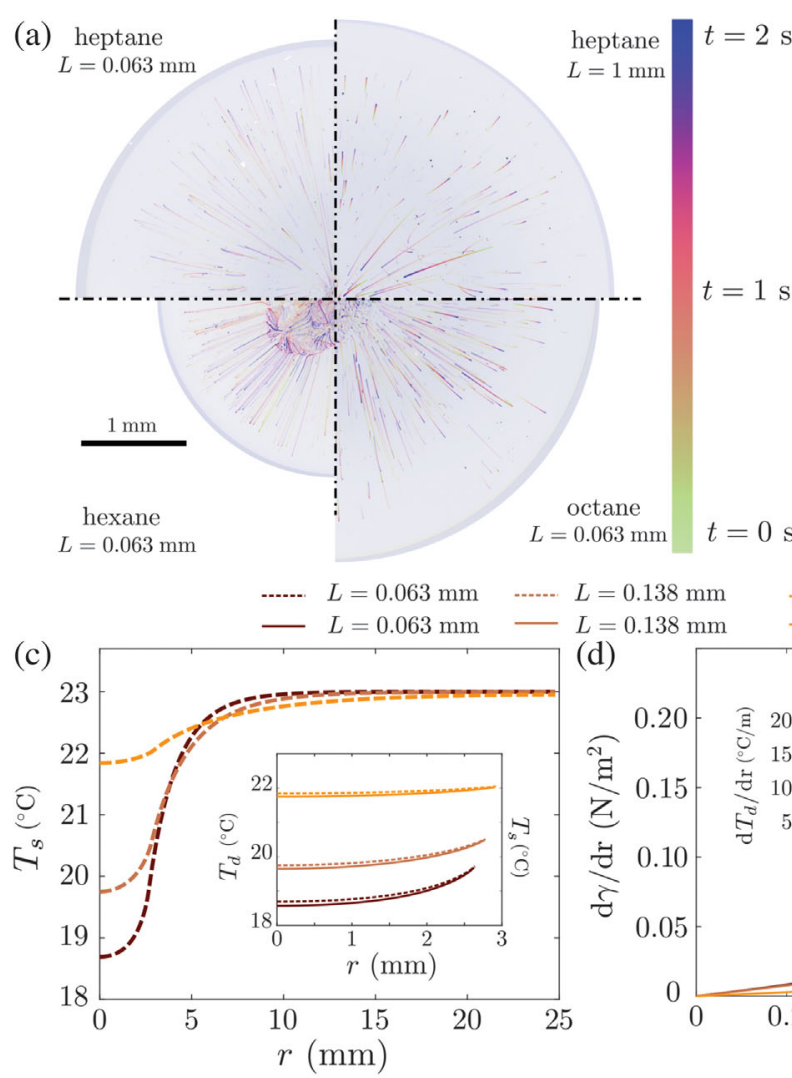

(b) $L$
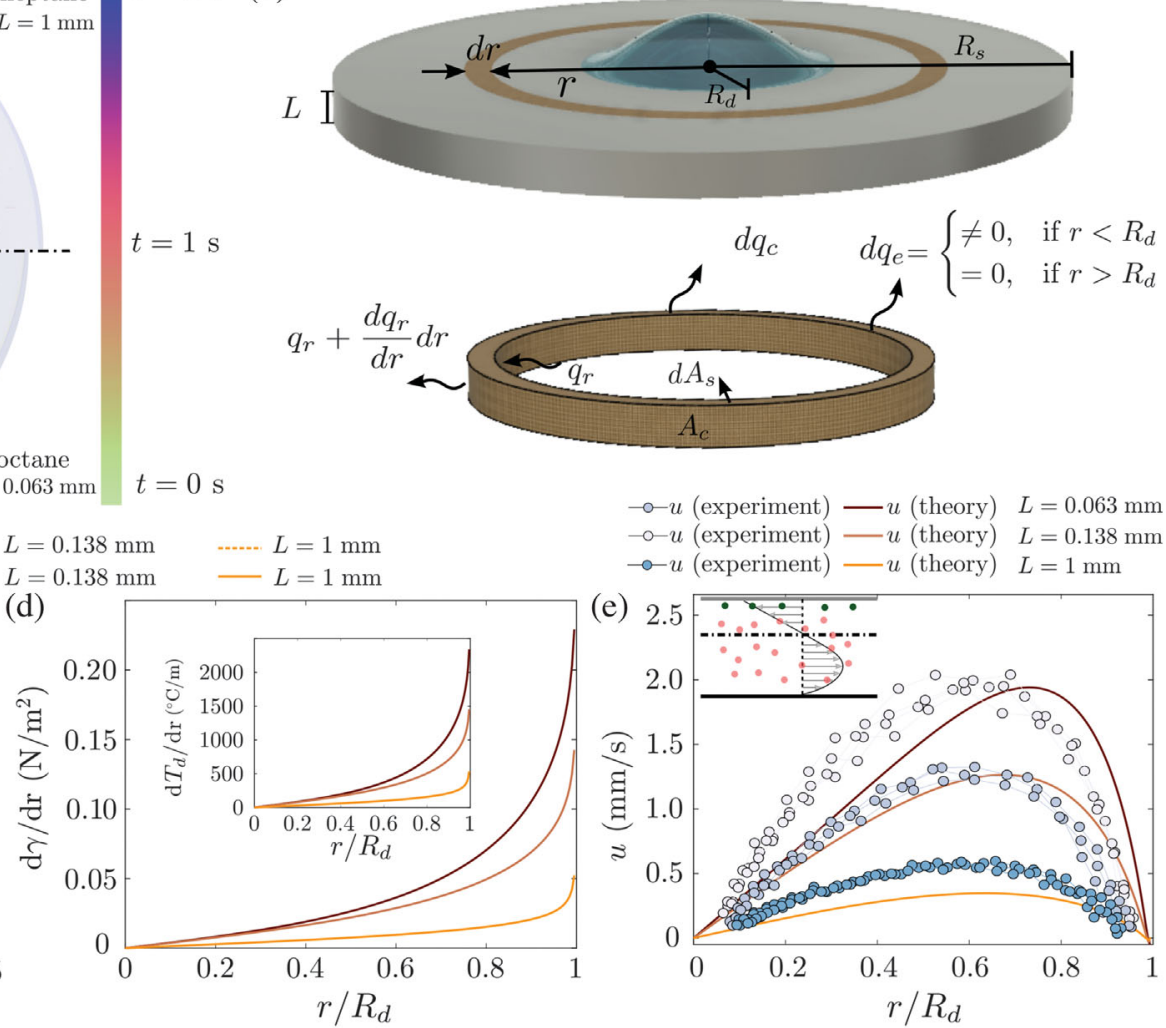

$\begin{aligned} L & =1 \mathrm{~mm} \\ -L & =1 \mathrm{~mm}\end{aligned}$

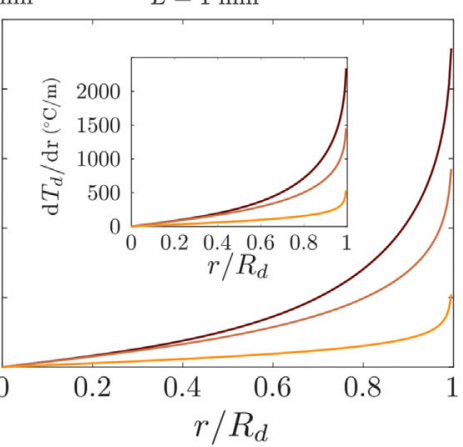

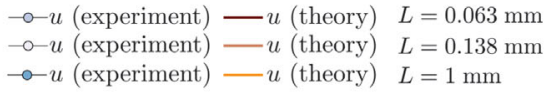

(1)

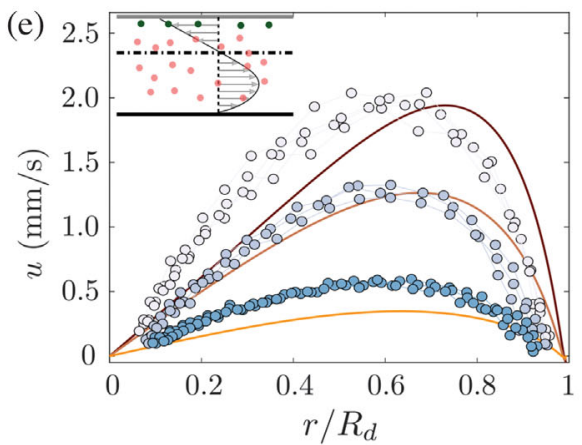

FIG. 3. Marangoni flow within evaporating alkane droplets on glass. (a) Time-lapse microscopy of tracer particles indicates flow direction and magnitude. Flow is fastest for hexane. Flow in heptane on thin glass is faster than heptane on thicker glass and, in octane tracer particles, sediment before fully circulating. (b) To formulate the governing equation, energy conservation is applied on a circular elements of radius $r$, thickness $d r$, and height $L$. We balanced energy by: conduction $q_{r}$ through the cross-sectional area $A_{c}$, free convection $d q_{c}$ through surface area $d A_{s}$, and removal by evaporation $d q_{e}$ within the droplet existence region. (c) Predicted radial substrate $\left[T_{s}\right.$, dashed, Eq. (4)] and droplet $\left[T_{d}\right.$, solid, Eq. (5), inset] temperature profiles, for heptane droplets on different glass thicknesses. (d) Surface tension gradients $d \gamma / d r$ are caused by temperature gradients $d T_{d} / d r$ (inset) for heptane droplets on different glass thicknesses. (e) Velocities $u$ of thermal Marangoni-induced flow for heptane droplets on different glass thicknesses. Experimental velocity was measured by tracking particles that followed streamlines near the droplet-vapor interface (indicated in green in inset), and theoretical values are from Eq. (6). 
where $\kappa_{\ell}$ is the liquid thermal conductivity, $T_{s}(r)$ is the substrate temperature, $T_{d}(r)$ is the droplet surface temperature, $h(r)$ is the droplet height, $\mathcal{L}$ is the latent heat of vaporization, and $j(r)$ is the local evaporative flux.

To determine the substrate temperature profile $T_{s}$, we modeled the substrate as a cylinder with radius $R_{s}$ and thickness $L$, Fig. 3(b). To formulate the governing equation, we applied conservation of energy to a circular element of radius $r$, thickness $d r$, and height $L$, assuming energy enters the element at rates $\left(d q_{r} / d r\right) d r$ by radial conduction and $d q_{c}$ by free convection. Additionally, the heat flow is driven by evaporation $d q_{e}=j(r) d A_{s}=$ $2 \pi r d r j(r)$ within the droplet region. For the evaporation term, considering a thin droplet in steady-state diffusionlimited evaporation, the expression for $j(r)$ can be approximated by Weber's disk [30]

$$
j(r)=\frac{j_{0}}{\sqrt{1-\left(r / R_{d}\right)^{2}}}, \quad j_{0}=\frac{2 D}{\pi R_{d}} \frac{M P_{\text {sat }}}{T R_{g}},
$$

where $R_{d}$ is the droplet radius, $D$ is the diffusion coefficient of vapor in air, $M$ is the molar mass, $P_{\text {sat }}$ is the saturated vapor pressure, $T$ is the temperature, and $R_{g}$ is the universal gas constant. Applying Fourier's law to convection and Newton's law to conduction and assuming quasisteady state, we arrive at the governing equation

$0 \leq r \leq R_{d}: \frac{d^{2} T_{s}^{i}}{d r^{2}}+\frac{1 d T_{s}^{i}}{r}-\frac{2 h_{c}}{d r}\left(T_{s}^{i}-T_{\infty}\right)=\frac{\mathcal{L} j_{0} / \kappa_{s} L}{\sqrt{\left(1-\left(r / R_{d}\right)^{2}\right)}}$,

$R_{d} \leq r \leq R_{s}: \frac{d^{2} T_{s}^{o}}{d r^{2}}+\frac{1 d T_{s}^{o}}{r}-\frac{2 h_{c}}{\kappa_{s} L}\left(T_{s}^{o}-T_{\infty}\right)=0$,

where superscripts $i$ and $o$ denote the region inside or outside of the droplet, respectively. $\kappa_{s}$ is the substrate thermal conductivity, $T_{\infty}$ is the ambient temperature, and $h_{c}$ is the heat convection coefficient.

The semianalytical solution for Eq. (3a), within the droplet region, can be found by assuming constant temperature in the convection term as $T_{s}^{i}(r)=T_{s}^{i}(0)$. The equation for the region outside of the droplet, Eq. (3b), is a Bessel differential equation with an analytical solution (Supplemental Material, Sec. II A [3]),

$$
\begin{aligned}
0 \leq r & \leq R_{d} \\
T_{s}^{i}(r) & =c_{1}+c_{2} \ln (r)+\frac{h_{c} r^{2}}{2 \kappa_{s} L}\left(T_{s}^{i}(0)-T_{\infty}\right) \\
& +\frac{\mathcal{L} j_{0} R_{d}^{2}}{\kappa_{s} L}\left(\ln \left(\sqrt{1-\left(r / R_{d}\right)^{2}}+1\right)-\sqrt{1-\left(r / R_{d}\right)^{2}}\right),
\end{aligned}
$$

$R_{d} \leq r \leq R_{s}: T_{s}^{o}(r)=c_{3} I_{0}\left(\sqrt{\frac{2 h_{c}}{\kappa_{s} L}} r\right)+c_{4} K_{0}\left(\sqrt{\frac{2 h_{c}}{\kappa_{s} L}} r\right)+T_{\infty}$.

Here $c_{1}, c_{2}, c_{3}$, and $c_{4}$ are constant values. $I_{0}$ and $K_{0}$ are the modified Bessel functions of order zero of the first and second kinds, respectively. Four boundary conditions are applied to solve for these constant values: symmetry of temperature at the center $d T_{s}^{i}(0) / d r=0$, continuity of temperature $T_{s}^{i}\left(R_{d}\right)=T_{s}^{o}\left(R_{d}\right)$, and continuity of heat flux $d T_{s}^{i}\left(R_{d}\right) / d r=d T_{s}^{o}\left(R_{d}\right) / d r$, at the interface between regions, and convection at the outer surface $-\kappa_{s} d T_{s}^{o}\left(R_{s}\right) / d r=$ $h_{c}\left(T_{s}^{o}\left(R_{s}\right)-T_{\infty}\right)$. The constants and unknown $T_{s}^{i}(0)$ are solved for iteratively, starting from an initial value of $T_{s}^{i}(0)=T_{\infty}$, (Supplemental Material, Sec. II B [3]).

Finally, liquid temperature along the droplet-vapor interface $T(d)$ can be obtained by substituting substrate temperature within a droplet region $T_{s}^{i}$ in Eq. (1),

$$
T_{d}(r)=T_{s}^{i}(r)-\frac{\mathcal{L} j_{0}}{\kappa_{\ell}}\left(h(r) / \sqrt{1-\left(r / R_{d}\right)^{2}}\right) .
$$

Equations (4) and (5) provide an analytical result that goes beyond previous work [16,20-22] and enables assessment of the magnitude of Marangoni flow, but we note this result relies on simplifications that may not be valid for all generic droplet scenarios (Supplemental Material, Sec. II C [3]).

Because of complex flows in hexane and tracer particle sedimentation in octane, we compared experimental to theoretical velocities for heptane. Applying Eqs. (4) and (5) to a heptane droplet gives temperature profiles of substrate and droplet-vapor interface for different substrate thicknesses, Fig. 3(c). From the radial temperature distribution, the radial temperature gradient, Fig. 3(d) inset, and surface tension gradient, Fig. 3(d), can be evaluated by considering $d \gamma / d r=(d \gamma / d T)(d T / d r)$ and assuming a parabolic droplet height profile [31]. These assumptions lead to a singularity at the contact line $d \gamma / d r\left(R_{d}\right)=\infty$ that might be eliminated by including the Kelvin effect [14] or lateral heat conduction within the liquid (Supplemental Material [3]).

To compute the flow velocity resulting from the Marangoni shear stress, for thin droplets, the lubrication approximation is applied to simplify Navier-Stokes equations in cylindrical coordinates, $\partial p / \partial r=\mu\left(\partial^{2} u / \partial z^{2}\right)$ [32]. Integrating twice, applying a no-slip boundary condition at the droplet-substrate interface $u(r, 0)=0$ and Marangoni stress at droplet-vapor interface $\tau(r)=\mu(\partial u / \partial z)=\partial \gamma / \partial r$ gives the velocity profile across the droplet height (Supplemental Material, Sec. II D [3]),

$$
u(r, z)=\frac{1}{2 \mu} \frac{\partial p}{\partial r} z^{2}+\frac{1}{\mu}\left(\frac{\partial \gamma}{\partial r}-\frac{\partial p}{\partial r} h(r)\right) z .
$$




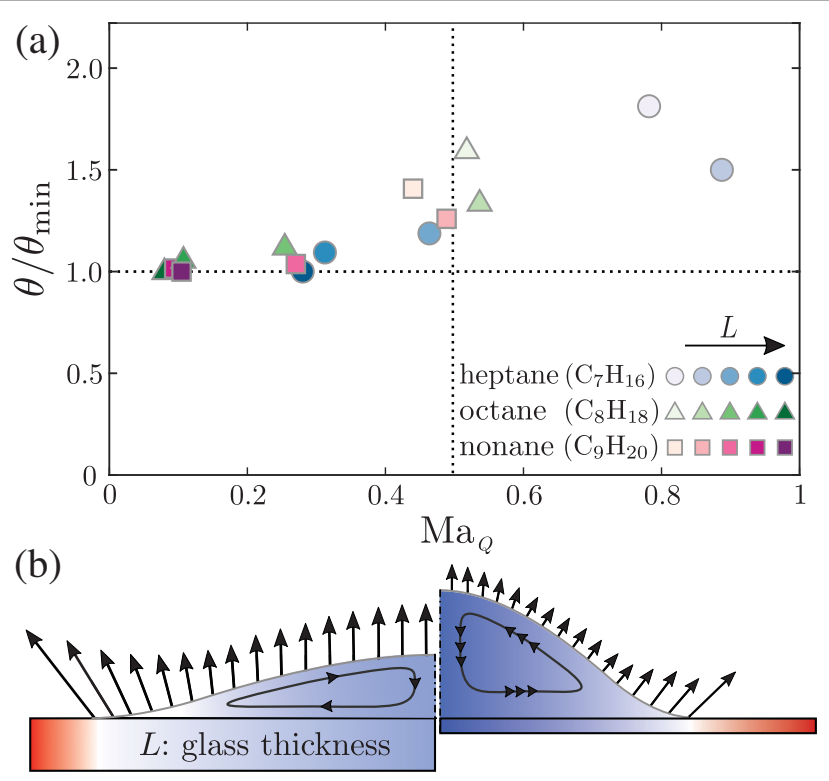

FIG. 4. Impact of Marangoni flows on droplet shape depends more on the substrate thickness than the evaporation rate. (a) Larger alternative Marangoni number $\mathrm{Ma}_{Q}$ indicates stronger Marangoni flow relative to potential capillary flow, which leads to a larger ratio in apparent contact angle to minimum apparent contact angle $\theta / \theta_{\min }$. Here $\theta_{\min }$ is the angle on the thickest glass, where the thermal Marangoni is the weakest. Shade indicates substrate thickness as in Fig. 2. (b) Schematic depiction showing evaporation of a droplet on thinner glass generating a larger temperature gradient that induces a stronger thermal Marangoni flow and a higher apparent contact angle despite slower evaporation.

For a pseudosteady-state droplet shape, the inward Marangoni flow is balanced by an outward pressuredriven capillary flow resulting in no net volumetric flux, $Q_{\text {net }}(r)=0=\int_{0}^{h(r)} 2 \pi r u(r, z) d z=2 \pi r\left[-\left(h^{3} / 3 \mu\right) \partial p / \partial r+\right.$ $\left.\left(h^{2} / 2 \mu\right) \partial \gamma / \partial r\right]$, which relates the pressure gradient to the surface tension gradient as: $\partial p / \partial r=(3 / 2 h)(\partial \gamma / \partial r)$.

Figure 3(e) shows the theoretical velocity a distance $r_{p}$ below the droplet-vapor interface, where $r_{p}$ is chosen as the particle radius to facilitate comparison to experiment. To extract particle velocities from the experiment at the liquidvapor interface [green circles on inset of Fig. 3(e)], only the speeds of tracer particles that travel nearly the entire distance from the droplet edge to center were measured. We find reasonable quantitative agreement between measured and predicted particle speeds across three different substrate thicknesses, Fig. 3(e), consistent with thermal Marangoni effects driving the observed flow.

To estimate whether thermal Marangoni effects need to be considered for droplet shape, we nondimensionalize the volumetric flux equation $Q_{\text {net }}$ and compute an alternative Marangoni number comparing volumetric flow rate from predicted Marangoni stress to potential capillarydriven flow, $\mathrm{Ma}_{Q}=-(3 / 2)(d \gamma / d T)\left(\Delta T / \gamma \theta^{2}\right)$, using our theoretically predicted droplet temperature along with radii and contact angles from experiment (see Supplemental Material, Sec. IIE for details [3] and related scaling in Ref. [16]). Figure 4(a) shows the ratio of measured contact angle $\theta$ to the minimum angle $\theta_{\min }$ (occurring on the thickest glass where the thermal Marangoni is the weakest). We find that above $\mathrm{Ma}_{Q} \approx 0.5$ the apparent contact angle begins increasing, Fig. 4(a). We interpret this to mean that if Marangoni flow is small relative to potential capillary flow for a given observed shape, then another effect such as the direct evaporation-driven mechanism primarily maintains the shape, but if Marangoni flow is large relative to potential capillary flows, it can distort the droplet and play a role in preventing spreading. This qualitatively agrees with a rough scaling comparing Marangoni and evaporative flow rates (Supplemental Material, Sec. II F [3]). We find that the impact of Marangoni flows on droplet shape depends more on the substrate thickness than the chain length of the alkane, a departure from scaling arguments made by other authors [9-12,17].

These results show thermal Marangoni effects can play a significant role in establishing droplet shape for volatile liquids on completely wetting thin substrates, Fig. 4(b). The similar flow structure between the thermal Marangoni contraction mechanism here and solutal Marangoni contraction of two-component droplets suggests that these droplets may share attributes. Indeed, recent work has shown droplet interactions between evaporating singlecomponent droplets [19,33-35], and this work adds another possibility to the growing list of interaction mechanisms for volatile droplets on high energy surfaces.

This work was supported by Rowland Institute at Harvard University. We thank Michael Burns, Masao Doi, and Xingkun Man for helpful discussions.

* Corresponding author. njc222@cornell.edu

[1] T. Young, Phil. Trans. R. Soc. London 95, 65 (1805).

[2] P. G. De Gennes, F. Brochard-Wyart, and D. Quéré, Capillarity and Wetting Phenomena: Drops, Bubbles, Pearls, Waves (Springer, New York, 2013).

[3] See Supplemental Material at http://link.aps.org/ supplemental/10.1103/PhysRevLett.127.024502 for material properties, which includes Refs. [4-6].

[4] H. Watanabe and D. Seong, Int. J. Thermophys. 23, 337 (2002).

[5] G. F. Carruth and R. Kobayashi, J. Chem. Eng. Data 18, 115 (1973).

[6] M. J. Tang, M. Shiraiwa, U. Pöschl, R. A. Cox, and M. Kalberer, Atmos. Chem. Phys. 15, 5585 (2015).

[7] L. H. Tanner, J. Phys. D Appl. Phys. 12, 1473 (1979).

[8] L. M. Hocking, Phys. Fluids 7, 2950 (1995).

[9] S. J. S. Morris, J. Fluid Mech. 432, 1 (2001).

[10] M. Cachile, O. Benichou, C. Poulard, and A. M. Cazabat, Langmuir 18, 8070 (2002). 
[11] C. Poulard, G. Guéna, A. M. Cazabat, A. Boudaoud, and M. Ben Amar, Langmuir 21, 8226 (2005).

[12] K. S. Lee, C. Y. Cheah, R. J. Copleston, V. M. Starov, and K. Sefiane, Colloids Surf. A Physicochem. Eng. Asp. 323, 63 (2008).

[13] A. Y. Rednikov, S. Rossomme, and P. Colinet, Multiphase science and technology 21, 213 (2009).

[14] J. Eggers and L. M. Pismen, Phys. Fluids 22, 112101 (2010).

[15] S. J. S. Morris, J. Fluid Mech. 739, 308 (2014).

[16] Y. Tsoumpas, S. Dehaeck, A. Rednikov, and P. Colinet, Langmuir 31, 13334 (2015).

[17] E. Jambon-Puillet, O. Carrier, N. Shahidzadeh, D. Brutin, J. Eggers, and D. Bonn, J. Fluid Mech. 844, 817 (2018).

[18] Y. Tsoumpas, S. Dehaeck, M. Galvagno, A. Rednikov, H. Ottevaere, U. Thiele, Uwe, and P. Colinet, Langmuir 30, 11847 (2014).

[19] H. Sadafi, S. Dehaeck, A. Rednikov, and P. Colinet, Langmuir 35, 7060 (2019).

[20] W. D. Ristenpart, P. G. Kim, C. Domingues, J. Wan, and H. A. Stone, Phys. Rev. Lett. 99, 234502 (2007).

[21] X. Xu, J. Luo, and D. Guo, Langmuir 26, 1918 (2010).

[22] H. Hu and R. G. Larson, Langmuir 21, 3972 (2005).

[23] F. Girard, M. Antoni, and K. Sefiane, Langmuir 24, 9207 (2008).

[24] W. D. Bascom, R. L. Cottington, and C. R. Singleterry, Adv. Chem. 43, 355 (1964).
[25] M. A. Hack, W. Kwieciński, O. Ramírez-Soto, T. Segers, S. Karpitschka, E. S. Kooij, and J. H. Snoeijer, Langmuir 37, 3605 (2021).

[26] N. J. Cira, A. Benusiglio, and M. Prakash, Nature (London) 519, 446 (2015).

[27] S. Karpitschka, F. Liebig, and H. Riegler, Langmuir 33, 4682 (2017).

[28] R. L. Cottington, C. M. Murphy, and C. R. Singleterry, Adv. Chem. 43, 341 (1964).

[29] C. Allain, D. Ausserre, and F. Rondelez, J. Colloid Interface Sci. 107, 5 (1985).

[30] H. Weber, J. Reine Angew. Math. 75 (1873).

[31] D. Bonn, J. Eggers, J. Indekeu, J. Meunier, and E. Rolley, Rev. Mod. Phys. 81, 739 (2009).

[32] C. Diddens, J. G. Kuerten, C. W. M. Van der Geld, and H. M. A. Wijshoff, J. Colloid Interface Sci. 487, 426 (2017).

[33] H. Teshima, S. Misra, K. Takahashi, and S. K. Mitra, Langmuir 36, 5096 (2020).

[34] Y. Wen, P. Y. Kim, S. Shi, D. Wang, X. Man, M. Doi, and T. P. Russell, Soft Matter 15, 2135 (2020).

[35] M. Wu, Y. Di, X. Man, and M. Doi, Langmuir 35, 14734 (2019).

Correction: The previously published Fig._3(c) contained errors in the $x$-axis numbers and has been set right. 